\title{
Impact of stopping therapy during the SARS-CoV-2 pandemic in persons with lymphoma
}

\author{
Shenmiao Yang ${ }^{1} \cdot$ Dong Dong $^{2} \cdot$ Hongfei Gu$^{3} \cdot$ Robert Peter Gale $^{4}\left(\mathbb{D} \cdot J_{\text {J }} \mathrm{Ma}^{5} \cdot\right.$ Xiaojun Huang $^{1}$
}

Received: 17 September 2020 / Accepted: 10 October 2020 / Published online: 19 October 2020

c) Springer-Verlag GmbH Germany, part of Springer Nature 2020

\begin{abstract}
Introduction The severe acute respiratory syndrome-2 (SARS-CoV-2) pandemic disrupted medical care for persons with cancer including those with lymphoma. Many professional societies recommend postponing, decreasing, or stopping anticancer therapy in selected persons during the pandemic. Although seemingly sensible, these recommendations are not evidence-based and their impact on anxiety and health-related quality-of-life (HRQoL) is unknown.

Methods We surveyed 2532 subjects including 1060 persons with lymphoma, 948 caregivers, and 524 normals using a purposed-designed questionnaire on a patient organization website. Respondents also completed the Zung Self-Rating Anxiety and patient respondents, the EORTC QLQ-C30 instruments to quantify anxiety, and HRQoL. We also evaluated caregiver support and an online education programme of the Chinese Society of Clinical Oncology (CSCO). Data of HRQoL from a 2019 pre-pandemic online survey of 1106 persons with lymphoma were a control.

Results 33\% (95\% confidence interval [CI] 30, 36\%) of lymphoma patients and 31\% (28, 34\%) of caregivers but only $21 \%$ $(17,24 \%)$ of normals had any level of anxiety (both pair-wise $P<0.001)$. Among lymphoma respondents, physical exercise and better caregiver support were associated with less anxiety, whereas female sex, receiving therapy, and reduced therapy intensity were associated with more anxiety. Paradoxically, lymphoma respondents during the pandemic had better HRQoL than pre-pandemic controls. Reduced therapy intensity was associated with worse HRQoL, whereas respondents who scored caregiver support and the online patient education programme high had better HRQoL.

Conclusion During the SARS-CoV-2 pandemic, lymphoma patients and their caregivers had significantly higher incidences of anxiety compared with normals. Lymphoma respondents reported better HRQoL compared with pre-pandemic controls. Reduced therapy intensity in persons with cancer may have unanticipated adverse effects on anxiety and HRQoL. Regular and intense support by caregivers and online education programmes alleviate anxiety and improve HRQoL.
\end{abstract}

Keywords Anxiety $\cdot$ Health related quality-of-life $\cdot$ Lymphoma $\cdot$ SARS-CoV-2 pandemic $\cdot$ COVID-19

\section{Introduction}

Jun Ma and Xiaojun Huang have contributed equally to this work.

Electronic supplementary material The online version of this article (https://doi.org/10.1007/s00432-020-03426-0) contains supplementary material, which is available to authorized users.

Xiaojun Huang

huangxiaojun@bjmu.edu.cn

1 Peking University Peoples Hospital, Peking University Institute of Hematology, National Clinical Research Center for Hematologic Disease, No.11, Xizhimen Nan Street, Beijing 100044, China

2 JC School of Public Health and Primary Care, Chinese University of Hong Kong, Hong Kong SAR, China
The severe acute respiratory virus coronavirus-2 (SARS$\mathrm{CoV}-2$ ) pandemic caused major changes also in health care support for patients with cancer worldwide (Maringe et al. 2020; Shah et al. 2019; Sud et al. 2020; van de Haar et al.

3 Chinese Lymphoma Patients Organization, House086, Beijing, China

4 Haematology Research Centre, Department of Immunology and Inflammation, Imperial College London, London, UK

5 Harbin Institute of Hematology and Oncology, Harbin, China 
2020). Recommendations from many professional societies suggest an individualized decision-making (Al-Shamsi et al. 2020; Di Ciaccio et al. 2020; ESMO 2020; Ismael et al. 2020). The general thrust is to reduce therapy intensity in patients with cancer with concerns that cancer and treatment may lead to a higher risk of infections and worse coronavirus infectious disease-2019 (COVID-19) outcomes.

We selected lymphoma as a frequent type of cancer and sought to investigate how the pandemic and typical interventions might impact on levels of anxiety among patients and their caregivers as well as how they affected patients' healthrelated quality-of-life (HRQoL). Therefore, we did an online survey using the platform of the Chinese lymphoma patient organization (House086). Patients and caregivers completed a questionnaire and standardized evaluation instruments to quantify levels of anxiety and HRQoL. The questionnaire and anxiety instrument were also completed by normals using the WeChat, a messaging and social media mobile app platform frequently used in China. We found that the prevalence of anxiety in lymphoma patient and caregiver respondents during the SARS-CoV-2 pandemic was significantly higher compared with normal. Unexpectedly, patient HRQoL during the pandemic was better compared with a propensity score matched pre-pandemic cohort. Better caregiver support was associated with less anxiety and better HRQoL. Access to an Internet-based lymphoma patient support platform and an education programme improved HRQoL. Several of these co-variates are actionable and may help to alleviate patients' concerns caused by SARS-CoV-2.

\section{Materials and methods}

\section{Study participants and study conduct}

We conducted a cross-sectional survey of lymphoma patients and their caregivers regarding the level of anxiety and patients' HRQoL between 17 and 19th April, 2020, using House 086 as the distribution platform (Supplement 1). Controls for the anxiety instrument were persons with no association with lymphoma patients or hospitals invited to participate in an online WeChat survey. Data from a HRQoL nationwide cross-sectional survey of lymphoma patients in 2019 were used to find matching cases as a pre-pandemic control cohort. The 2019 lymphoma survey included 4068 Chinese with all sub-types of lymphoma from which 1106 patients matched on sex, age, education level, and sub-type of lymphoma and therapy type were extracted.

Sample size was calculated as $n=(z)^{2} p(1-p) / d^{2}$, in which $z=1.96$ for a level $(\alpha)$ of confidence of $95 \%$. Tolerated margin of error was 0.05 . The prevalence of clinically important anxiety in the Chinese population is reported as 29-35\% (Huang and Zhao 2020; Wang et al. 2020).
Minimum number of the qualified questionnaires was estimated as 350 .

An online questionnaire collected data on: (1) demographics; (2) lymphoma-related data; (3) impact of the pandemic on health care-related activities; (4) hours of mobile phone use; (5) online patient-assistance resources; (6) quality of caregiver support (10-point scale); and (7) quality of the CSCO online education programme (10-point scale). To quantify anxiety of lymphoma patients, caregivers, and normals, we used the Zung Self-Rating Anxiety Scale (SAS) (Chinese version) (Liu et al. 1997; Zung 1971; ZY 1984). We used the EORTC QLQ-C30 (v.3; Chinese version) to quantify lymphoma patients' HRQoL (Aaronson et al. 1993; Zhao and Kanda 2000).

The study was approved by the Ethics Committees of Peking University Peoples' Hospital according to tenets of the Declaration of Helsinki (Register number 2020PHB173). Electronic informed consent was obtained from all respondents who could withdraw at any time during the survey without prejudice.

\section{Statistical analysis}

Descriptive statistics were used for the demographic, social, and lymphoma-related co-variates. Anxiety index (AI) was calculated according to the Zung SAS, a rating instrument for anxiety disorders. Based on extensive validated data, an $\mathrm{AI}<50$ is defined as normal, 50-59, $\mathrm{minimal} / \mathrm{moder}-$ ate, 60-69, marked/severe, and $\geq 70$, extreme anxiety in the Chinese population with an internal consistency reliability of 0.66-0.80 and the Cronbach $\alpha$ of 0.87 (Minglu et al. 2020; Shao et al. 2020). Scores of five functioning scales (physical, role, emotional, cognitive, and social functioning), eight symptom scales (fatigue, nausea/vomiting, pain, dyspnea, sleep disturbances, appetite loss, constipation, and diarrhea), fiscal impact, and overall HRQoL were calculated using the EORTC QLQ-C30 instrument (Aaronson et al. 1993).

Lymphoma respondents from this study were matched with respondents to the 2019 pre-pandemic study on covariates including age, education level, lymphoma sub-type, and therapy by the nearest neighbor matching method with $\mathrm{R}$ packages "MatchIt" at a 1:1 ratio. Standardized mean difference was calculated for each of the co-variates between the cohorts before and after matching to assess matching quality. An absolute standardized difference of $>20 \%$ denotes meaningful co-variate imbalance (Supplement 2).

We used the Independent-Samples t test to compare groups of continuous variables and one-way analysis of variance (ANOVA) and LSD to analyze differences among cohorts and each paired cohort. Chi-square test was used to analyze categorical co-variates. We used a multi-variable analysis with binary logistic regression to identify risk factors of anxiety. The Kendall tau-b correlation was used to 
evaluate risk factors of HRQoL. Tests were two-sided and $P \leq 0.05$ was considered significant. Statistical analyses were performed with SPSS 12.0 (IBM SPSS Statistics, New York, US).

\section{Results}

We received 2745 responses from subjects in 32 Chinese provinces, autonomous regions, centrally administered municipalities, and special administrative regions identified by internet protocol (IP) addresses. 166 questionnaires, incomplete or completed in $<1 \mathrm{~min}$ or $>60 \mathrm{~min}$, were excluded from further analyses. 94 percent of questionnaires were evaluable.

\section{Respondent co-variates}

$1106(43 \%)$ of the 2578 respondents were lymphoma patients, 948 (37\%), caregivers and $524(20 \%)$, normals (Table 1). 1031 respondents (40\%) were male and 2313 (90\%), 20-60 years. 495 (45\%) patient respondents were 20-39 years, 477 (43\%) were $40-59$ years, and $110(10 \%)$ were $\geq 60$ years. There was a discordance between patients' age reported by patients and caregivers (Table 2). 1912 (74\%) respondents were college graduates or received other higher education. 88 (3\%) lived in Hubei province with Wuhan as the capital city known to be the first area on lockdown from 23rd January to 8th April in China. 275 (11\%) were living outside their usual residence. 15 reported that they were infected with SARS-CoV-2 and another 19, their friends or relatives were infected with SARS-CoV-2.

Distribution of lymphoma types reported by patient and caregiver respondents was similar to lymphoma distribution data from China (Cao et al. 2018; Sun et al. 2012; Yang et al. 2011). 654 (59\%) patient and 700 (74\%) caregiver respondents reported an aggressive lymphoma $(P<0.001$; Table 2$)$. 966 (47\%) patient respondents were on-therapy, parenteral in 707 (34\%) and oral in 259 (13\%). 1088 (53\%) patient respondents were under medical supervision with no current therapy. $819(47 \%)$ receive in-hospital $(40 \%)$ or outpatient (7\%) therapy.

1155 (56\%) patients changed the hospital they had been visiting for routine monitoring and/or therapy during the SARS-CoV-2 pandemic. 192 (9\%) changed to a therapy of lower intensity. 89 (4\%) switched to oral anti-lymphoma drugs, 259 (13\%) delayed scheduled parenteral therapy, and 761 (37\%) delayed or postponed scheduled hospital visits. $482(24 \%)$ experienced reduced therapy intensity including fewer drugs, reduced drug doses, a switch from parenteral to oral drugs, and/or therapy delay or discontinuation. 1059 (52\%) reported no change of their medical activities including physician visits, exams, and/or therapy.

\section{Respondent concerns}

The most frequent concerns of patient respondents were their lymphoma $(N=603 ; 55 \%)$, SARS-CoV-2-infection $(N=547 ; 50 \%)$, and the inability to attend outpatient clinics $(N=429 ; 39 \%)$. The most frequent concerns for caregiver respondents related to the patient they were caring for were lymphoma $(N=595 ; 63 \%)$, therapy disruption $(N=397$; $42 \%)$, and SARS-CoV-2-infection $(N=392 ; 41 \%)$. The most frequent concerns of normal respondents were SARS-CoV2-infection risk to their family $(N=347 ; 66 \%)$, themselves $(N=308 ; 59 \%)$, and income loss $(N=198 ; 38 \%)$.

\section{Respondent anxiety}

Respondents with any level of anxiety (i.e., Zung score $>50$ ) were $33 \%$ (95\% confidence interval [CI] 30, 36\%) for lymphoma patients, $31 \%(28,34 \%)$ for caregivers, and $21 \%$ (17, $24 \%$; three-cohort comparison: $P<0.001)$ for normal individuals. Pair-wise comparisons showed incidence of anxiety was similar in patients and caregiver respondents $(P=0.29)$ but higher compared with normals (both $P<0.001$ ). Severity of anxiety was similar in the three cohorts. Among persons with anxiety, minimal/moderate severity levels (score $>49$ ) were $77 \%, 70 \%$, and $72 \%$, marked/severe anxiety (score $>59$, $19 \%, 23 \%$, and $22 \%$ and extreme anxiety (score $>69), 4 \%$, $7 \%$, and $6 \%(P=0.22)$.

We evaluated co-variates associated with anxiety in respondents (Table 3). No SARS-CoV-2 infection (Hazard Ratio $[\mathrm{HR}]=0.15(0.041,0.53 ; P=0.003)$, being a normal versus a patient or caregiver $(\mathrm{HR}=0.53[0.42$, 0.68 ]; $P<0.001)$, physical exercise $(\mathrm{HR}=0.60(0.49$, $0.73 ; P<0.001)$, higher education level $(\mathrm{HR}=0.77(0.63$, 0.93; $P=0.007)$, increase of $>2 \mathrm{~h} /$ day of mobile phone use (HR $=0.83$ [0.70, 0.99]; $P=0.042$ ), and higher family support score $(\mathrm{HR}=0.93[0.89,0.96] ; P=0.001)$ were associated with less anxiety.

Patient respondents not hospitalized during the pandemic but before the survey had less anxiety of any severity compared with hospitalized respondents $(\mathrm{HR}=0.62[0.48,0.81]$; $P=0.004)$. Frequency of marked/severe or extreme severity was also increased in hospitalized versus not hospitalized patient respondents (HR $=0.62$ [0.40, 0.98]; $P=0.042)$. Among patient respondents not hospitalized during the pandemic but before the survey, persons with a higher family support score had lower incidence of anxiety $(\mathrm{HR}=0.90$ $[0.85,0.96] ; P=0.002)$. Among patient respondents hospitalized during the pandemic, but before the survey, there was no correlation between incidence of anxiety and caregiver support score $(\mathrm{HR}=0.97[0.86,1.08] ; P=0.54)$.

In multi-variable analyses, we found more patients receiving therapy (HR $=1.43[1.08,1.89] ; P=0.012)$, those with reduced therapy intensity $(\mathrm{HR}=1.59[1.14,2.21] ; P=0.006)$ 
Table 1 Co-variates of all respondents

\begin{tabular}{|c|c|c|c|}
\hline & \multicolumn{3}{|l|}{ No. $(\%)$} \\
\hline & Patients $(n=1106)$ & Caregivers $(n=948)$ & Normals $(n=524)$ \\
\hline Male & $482(44)$ & $293(31)$ & $256(49)$ \\
\hline \multicolumn{4}{|l|}{ Age, years } \\
\hline $18-20$ & $24(2)$ & $8(1)$ & $70(13)$ \\
\hline $20-39$ & $495(45)$ & $544(57)$ & $280(53)$ \\
\hline $40-59$ & $477(43)$ & $371(39)$ & $146(28)$ \\
\hline $60-79$ & $108(10)$ & $25(3)$ & $27(5)$ \\
\hline$\geq 80$ & $2(1)$ & 0 & $1(1)$ \\
\hline \multicolumn{4}{|l|}{ Education } \\
\hline Primary school & $25(2)$ & $18(2)$ & $6(1)$ \\
\hline Middle/high school & $331(30)$ & $195(21)$ & $91(17)$ \\
\hline College/university & $682(62)$ & $639(67)$ & $379(72)$ \\
\hline$\geq$ Postgraduate & $68(6)$ & $96(10)$ & $48(9)$ \\
\hline SARS-CoV-2-infection & $5(1)$ & $10(1)$ & 0 \\
\hline SARS-CoV-2-infection in family or friends & $5(1)$ & 11(1) & $3(1)$ \\
\hline Hubei resident & $52(5)$ & $30(3)$ & $6(1)$ \\
\hline \multicolumn{4}{|l|}{ Physical exercise } \\
\hline No & $214(19)$ & $251(27)$ & $130(25)$ \\
\hline Yes & $892(80)$ & $697(74)$ & $394(75)$ \\
\hline \multicolumn{4}{|l|}{ Mobile phone use pre-pandemic, h/day } \\
\hline$<3$ & $551(50)$ & $549(58)$ & $268(51)$ \\
\hline $4-5$ & $361(33)$ & $263(28)$ & $157(31)$ \\
\hline $6-7$ & $126(11)$ & $90(10)$ & $57(11)$ \\
\hline$\geq 8$ & $68(6)$ & $46(5)$ & $42(8)$ \\
\hline \multicolumn{4}{|l|}{ Mobile phone $\mathrm{h} /$ day during pandemic } \\
\hline$<3$ & $358(32)$ & $308(33)$ & $149(28)$ \\
\hline $4-5$ & $409(37)$ & $376(40)$ & $181(35)$ \\
\hline $6-7$ & $204(18)$ & $167(18)$ & $98(19)$ \\
\hline$\geq 8$ & $135(12)$ & $97(10)$ & $96(18)$ \\
\hline \multicolumn{4}{|l|}{ Concerns } \\
\hline SARS-CoV-2-infection & $547(50)$ & $392(36)$ & $308(59)$ \\
\hline SARS-CoV-2 infection of family/ friends & $214(19)$ & $146(15)$ & $347(66)$ \\
\hline Family separation & $28(3)$ & $22(2)$ & $180(34)$ \\
\hline Income loss & $204(18)$ & $151(16)$ & $198(38)$ \\
\hline Lymphoma & $603(55)$ & $595(63)$ & - \\
\hline \multicolumn{4}{|l|}{ Read online or obtained information } \\
\hline SARS-CoV-2 infection or/and COVID-19 & $946(86)$ & $822(87)$ & $471(90)$ \\
\hline Lymphoma & $572(84)$ & $539(91)$ & - \\
\hline Entertainment & $610(55)$ & $370(39)$ & $410(78)$ \\
\hline Work & $209(19)$ & $228(24)$ & $252(48)$ \\
\hline \multicolumn{4}{|l|}{ Family support } \\
\hline Score, mean (SD) & $8.84(2.16)$ & $8.93(1.97)$ & $8.25(2.10)$ \\
\hline Anxiety index, mean (SD) & $45.8(9.4)$ & $46.0(9.8)$ & $42.6(9.9)$ \\
\hline \multicolumn{4}{|l|}{ Clinical interpretation of AI } \\
\hline Normal $(<50)$ & $793(67)$ & $654(69)$ & $416(79)$ \\
\hline Minimal/moderate (50-59) & $284(26)$ & $207(22)$ & $78(15)$ \\
\hline Marked/ever anxiety (60-69) & $70(6)$ & $67(7)$ & $24(5)$ \\
\hline Extreme $(\geq 70)$ & $13(1)$ & $20(2)$ & $6(1)$ \\
\hline
\end{tabular}


Table 2 Co-variates of lymphoma patient respondents

\begin{tabular}{|c|c|c|c|}
\hline & \multicolumn{3}{|l|}{ No. $(\%)$} \\
\hline & Patients $(n=1106)$ & Caregivers $(n=948)$ & Both $(n=2054)$ \\
\hline Male & $482(44)$ & $541(57)$ & $1023(50)$ \\
\hline \multicolumn{4}{|l|}{ Age, years } \\
\hline$<20$ & $24(2)$ & $96(10)$ & $120(6)$ \\
\hline $20-39$ & $495(45)$ & $184(19)$ & $679(33)$ \\
\hline $40-59$ & $477(43)$ & $368(39)$ & $845(41)$ \\
\hline $60-79$ & $108(10)$ & $291(31)$ & 399 (19) \\
\hline$\geq 80$ & $2(1)$ & $9(1)$ & $11(1)$ \\
\hline \multicolumn{4}{|l|}{ Lymphoma type } \\
\hline Hodgkin & $89(8)$ & $70(7)$ & $159(8)$ \\
\hline \multicolumn{4}{|l|}{ Non-Hodgkin } \\
\hline Indolent & $361(33)$ & $174(18)$ & $535(26)$ \\
\hline Follicular & $210(19)$ & $86(9)$ & $296(14)$ \\
\hline Marginal zone & $64(6)$ & $39(4)$ & $103(5)$ \\
\hline CLL/SLL & $69(6)$ & $31(3)$ & $100(5)$ \\
\hline Lymphoplasmacytic & $3(1)$ & $3(1)$ & $6(1)$ \\
\hline Other & $15(1)$ & $15(2)$ & $30(2)$ \\
\hline Aggressive & $654(59)$ & $700(74)$ & $1354(66)$ \\
\hline Diffuse large B cell & $405(37)$ & $413(44)$ & $818(40)$ \\
\hline Burkitt & $26(2)$ & $30(3)$ & $56(3)$ \\
\hline Mantle cell & $30(3)$ & $39(4)$ & $69(3)$ \\
\hline Extra-nodal NK/T cell & $66(6)$ & $61(6)$ & $127(6)$ \\
\hline Peripheral T cell, not otherwise specified & $32(3)$ & $46(5)$ & $78(4)$ \\
\hline Anaplastic large T cell & $43(4)$ & $46(5)$ & $89(4)$ \\
\hline Lymphoblastic & $37(3)$ & $46(5)$ & $83(4)$ \\
\hline Other $\mathrm{T}$ cell & $15(1)$ & $19(2)$ & $34(2)$ \\
\hline Unknown & $2(1)$ & $4(1)$ & $6(1)$ \\
\hline \multicolumn{4}{|l|}{ Stage } \\
\hline Early & $416(38)$ & $279(29)$ & $695(34)$ \\
\hline Advanced & $519(53)$ & $556(59)$ & $1075(52)$ \\
\hline Unknown & $99(9)$ & $113(12)$ & $212(10)$ \\
\hline \multicolumn{4}{|l|}{ On-therapy } \\
\hline No & $676(61)$ & $412(44)$ & $1088(53)$ \\
\hline Parenteral & $301(27)$ & $406(43)$ & $707(34)$ \\
\hline Oral & $129(12)$ & $130(14)$ & $259(13)$ \\
\hline Changed hospital & $633(57)$ & $522(55)$ & $1155(56)$ \\
\hline \multicolumn{4}{|l|}{ Change in medical activities } \\
\hline Delayed therapy & $124(11)$ & $135(14)$ & $259(13)$ \\
\hline Less intensive therapy & $82(7)$ & $110(12)$ & $192(9)$ \\
\hline Switched to oral drugs & $41(4)$ & $48(5)$ & $89(4)$ \\
\hline Delayed exams & $441(40)$ & $317(33)$ & $728(35)$ \\
\hline No change & $570(52)$ & $489(52)$ & $1059(52)$ \\
\hline Reduced intensity & $217(20)$ & $265(28)$ & $482(24)$ \\
\hline \multicolumn{4}{|l|}{ Achievement in online programme } \\
\hline Assistance in confirming lymphoma diagnosis & $208(31)$ & $215(36)$ & $423(33)$ \\
\hline Therapy assistance & $246(36)$ & $271(46)$ & $517(41)$ \\
\hline SARS-CoV-2 prevention or COVID-19 therapy & $163(24)$ & $133(23)$ & $296(23)$ \\
\hline Physician communication & $201(29)$ & $195(33)$ & $396(31)$ \\
\hline Patient/caregiver communication & $226(33)$ & $222(38)$ & $448(35)$ \\
\hline
\end{tabular}


Table 2 (continued)

\begin{tabular}{|c|c|c|c|}
\hline & \multicolumn{3}{|l|}{ No. $(\%)$} \\
\hline & Patients $(n=1106)$ & Caregivers $(n=948)$ & Both $(n=2054)$ \\
\hline Improved mood & $325(48)$ & $278(47)$ & $603(47)$ \\
\hline Increased confidence & $362(53)$ & $372(53)$ & $734(58)$ \\
\hline
\end{tabular}

and females $(\mathrm{HR}=1.33[1.02,1.72] ; P=0.034)$ had a higher incidence of anxiety, whereas physical exercise $(\mathrm{HR}=0.57$ [0.42, 0.77]; $P<0.001)$ and higher family support score $(\mathrm{HR}=0.92[0.87,0.98] ; P=0.006)$ were associated with lower incidences of anxiety (Table 3 ).

\section{Caregivers}

Caregiver respondents were knowledgeable of patients' diagnoses, stage and therapy, were aware of difficulties patients faced, and were enthusiastic to receive information about lymphoma-related medical aspects (Table 1). Patient respondents gave their caregivers a mean support score of $8.84 \pm 2.16(\mathrm{SD})$.

\section{Mobile phone use}

There was a large increase in mobile phone use during the SARS-CoV-2 pandemic in China. $12 \%(10,14 \%)$ of patient respondents, $10 \%(8,12 \%)$ of caregivers, and $18 \%(15,22 \%$; $P<0.001$ ) of normals used their mobile phones (the main source of Internet access in China) for $\geq 8 \mathrm{~h}$ per day versus $6 \%(5,8 \% ; P<0.001), 5 \%(4,6 \% ; P<0.001)$, and $8 \%$ $(6,11 \% ; P<0.001)$ before the pandemic. $40 \%(37,43 \%)$, $50 \%(46,53 \%)$, and $52 \%(47,56 \%)$ reported an increase in mobile phone use by $>2$ h daily during the pandemic. $86 \%$ $(83,88 \%), 87 \%(84,89 \%)$, and $90 \%(87,92 \%, P=0.052)$ of patient, caregiver, and normal respondents accessed pandemic news on their mobile phones. $42 \%$ (39, 45\%), $29 \%$ $(26,32 \%)$, and $53 \%(48,57 \% ; P<0.001)$ of patient, caregiver, and normal respondents reported to use their mobile phone for entertainment including reading novels, movies or TV. $75 \%(72,78 \%)$, and $84 \%(81,86 \% ; P<0.001)$ of patients and caregiver respondents reported reading news about lymphoma or participated in the CSCO lymphoma education programme.

\section{Internet-based CSCO programme}

$1275(62 \%)$ of patients and caregiver respondents reported participating in the CSCO education programme. $236(35 \%$ $[31,38 \%])$ of patient and $238(40 \%,[36,44 \%], P=0.037)$ of caregiver respondents participated in $\geq 3$ sessions. $53 \%$ (49, $57 \%)$ of patient and $63 \%(59,67 \%)$ of caregiver respondents reported increased confidence after participating in the programme $(P<0.001)$. The programme was scored $8.42 \pm 1.86(\mathrm{SD})$ by patient respondents and $8.47 \pm 1.93$ by caregiver respondents $(P=0.68)$.

\section{Quality-of-life}

We used the EORTC QLQ-C30 instrument to compare lymphoma patient respondents' HRQoL at the time of our study with that of Chinese lymphoma patients in a survey in 2019 after matching for sex, age, education level, lymphoma type, and therapy in a propensity score analysis (Supplement 2). Patient respondents had better HRQoL scores compared with controls. Physical, role, emotional, cognitive, social functioning, and general HRQoL were significantly better (all $P$ values $<0.05$ ). Nausea and vomiting, dyspnea, insomnia, constipation, and financial difficulties were milder (all $P$ values $<0.05$ ) compared with pre-pandemic controls (Table 4).

Next, we analyzed co-variates correlated with HRQoL. Increased daily mobile phone use and participation in the education programme were not correlated with HRQoL. In contrast, reduced therapy intensity was significantly associated with a worse general HRQoL and five worse functions, eight symptoms, and financial difficulties. Patient respondents who scored caregiver support high had a better general HRQoL, emotional function, cognitive function, and fewer or less severe insomnia and diarrhea. Patient respondents who scored the education programme high had better HRQoL including all five functions and fewer symptoms of nausea and vomiting, dyspnea, insomnia, and diarrhea (Supplement 3).

\section{Discussion}

The SAR-CoV-2 pandemic in China reduced lymphoma patients' access to medical care including out- and inpatient clinic and hospital visits and direct contact with medical personnel including doctors and nurses. It also decreased potential interactions with other lymphoma patients they might encounter in clinic or hospital settings. Because of travel restrictions, some patients had to switch their pointof-contact for medical care, for example, to a nearby clinic or hospital. Decreased blood testing and pharmacy access led to therapy modifications such as switching to oral drugs. 


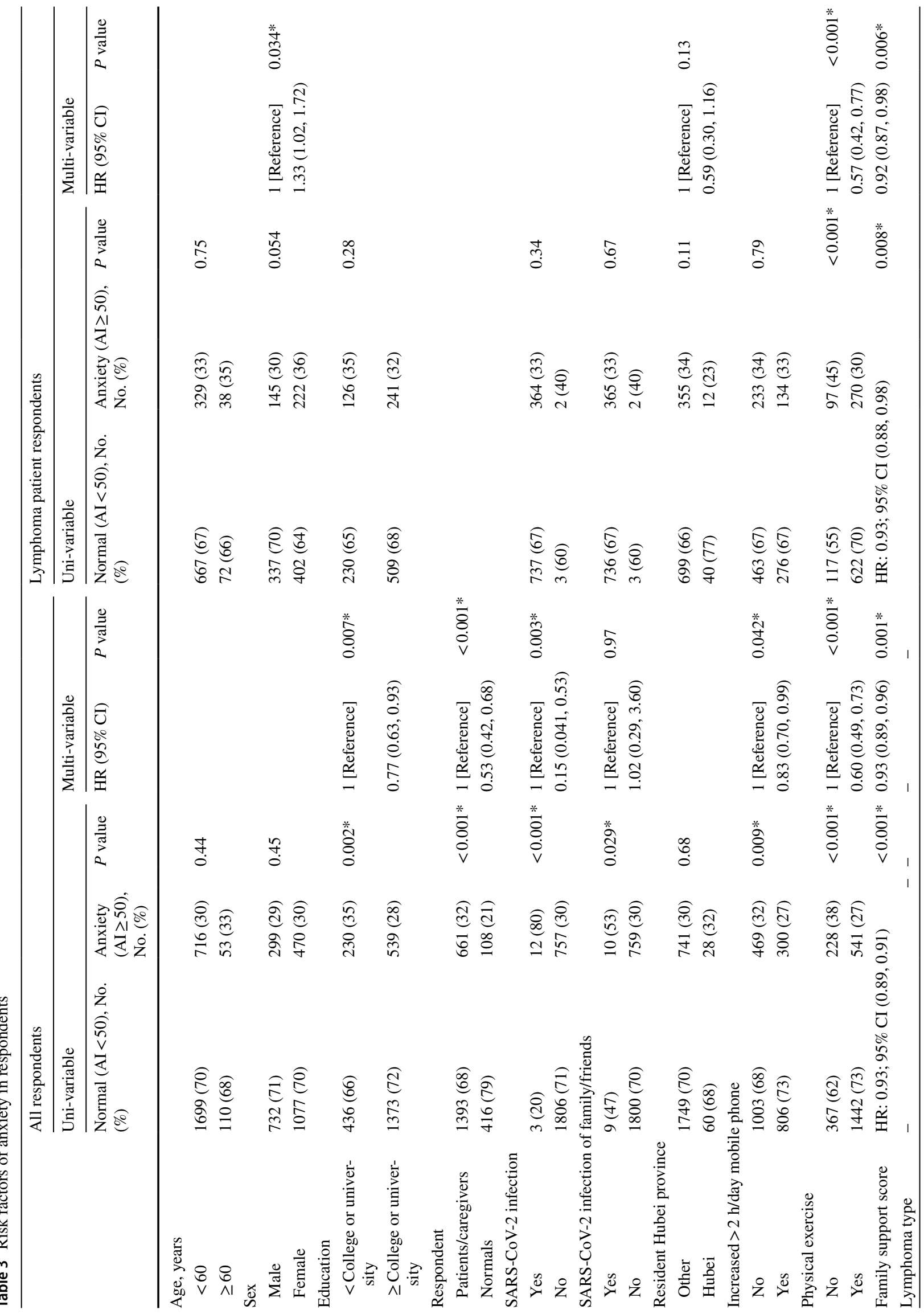




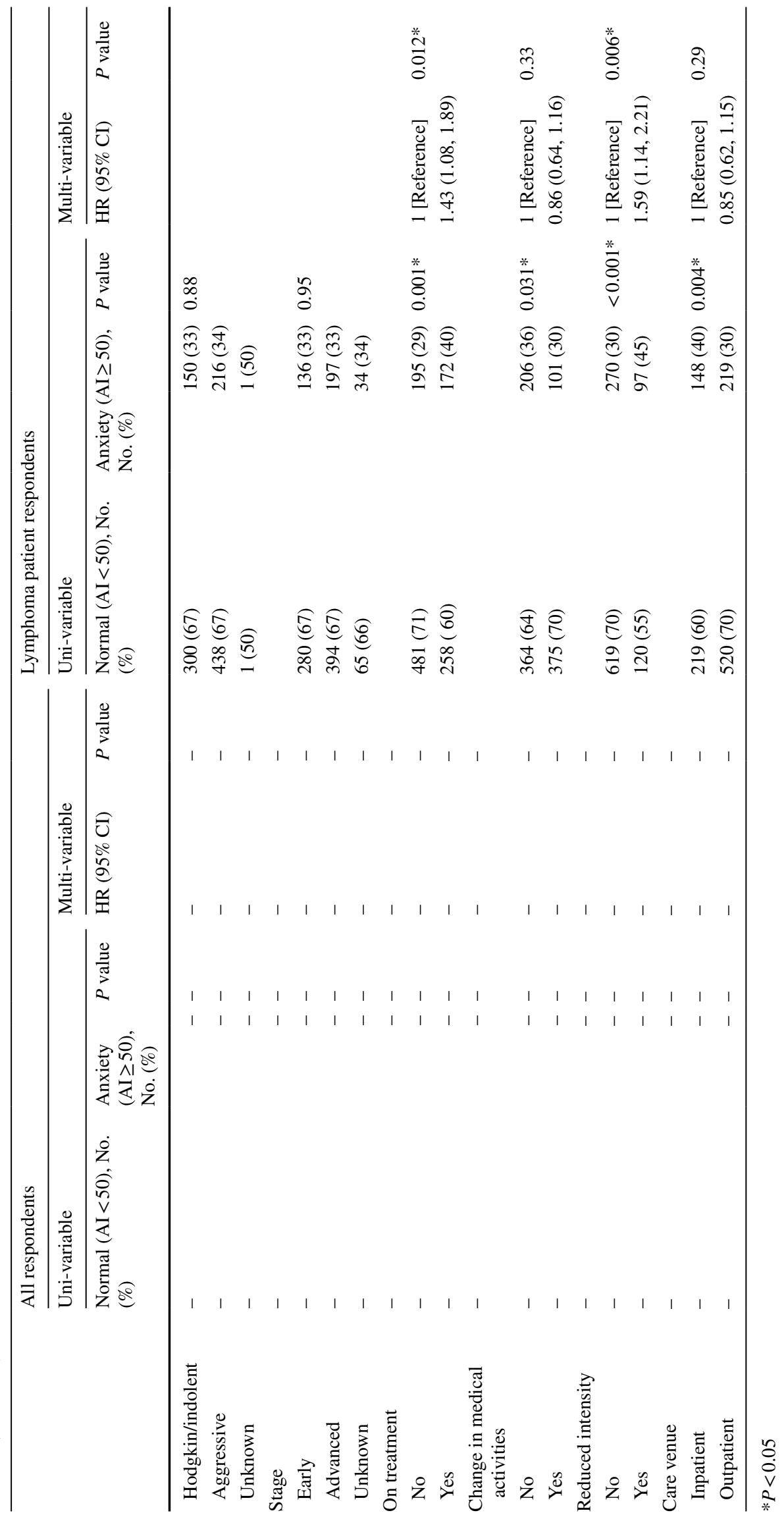


Table 4 HRQoL of lymphoma patients during the pandemic and prepandemic

\begin{tabular}{|c|c|c|c|}
\hline \multirow[t]{2}{*}{ HRQoL scale/item } & \multicolumn{2}{|c|}{ HRQoL of patients, mean (SD) } & \multirow[t]{2}{*}{$P$ value } \\
\hline & $\begin{array}{l}\text { In the } \\
\text { pandemic } \\
(n=1106)\end{array}$ & $\begin{array}{l}\text { In } 2019 \text { pre- } \\
\text { pandemic } \\
(n=1106)\end{array}$ & \\
\hline $\begin{array}{l}\text { Global health status/ } \\
\text { QoL }\end{array}$ & $70.1(21.4)$ & $59.7(23.1)$ & $<0.001 *$ \\
\hline \multicolumn{4}{|l|}{ Functional scale } \\
\hline Physical functioning & $81.9(16.5)$ & $79.2(18.1)$ & $0.025 *$ \\
\hline Role functioning & $81.3(24.1)$ & $73.7(27.3)$ & $<0.001 *$ \\
\hline Emotional functioning & $74.9(21.0)$ & $66.0(23.7)$ & $<0.001 *$ \\
\hline Cognitive functioning & $78.4(19.6)$ & $74.4(21.2)$ & $<0.001 *$ \\
\hline Social functioning & $62.3(28.6)$ & $49.3(30.1)$ & $<0.001 *$ \\
\hline \multicolumn{4}{|l|}{ Symptom scale/item } \\
\hline Fatigue & $35.9(22.1)$ & $41.6(22.7)$ & 0.81 \\
\hline Nausea and vomiting & $9.1(17.2)$ & $10.7(18.9)$ & $0.019 *$ \\
\hline Pain & $18.7(21.2)$ & $20.3(21.5)$ & 0.12 \\
\hline Dyspnea & $17.1(21.2)$ & $22.3(23.7)$ & $0.006^{*}$ \\
\hline Insomnia & $28.6(29.0)$ & $31.6(28.5)$ & $<0.001 *$ \\
\hline Appetite loss & $16.2(23.3)$ & $21.2(24.6)$ & 0.89 \\
\hline Constipation & $15.1(5.22)$ & $16.4(24.6)$ & $<0.001 *$ \\
\hline Diarrhea & $11.9(19.3)$ & $13.8(20.5)$ & 0.072 \\
\hline Financial difficulties & $46.3(34.6)$ & $62.2(34.4)$ & $<0.001 *$ \\
\hline
\end{tabular}

$* P<0.05$

These impacts of the pandemic occurred globally (Chen-See 2020; Triggle 2020).

Because these co-variates are important determinants of anxiety and HRQoL in lymphoma patients, we sought to quantify these and to determine the impact, if any, of alternative support resources such as caregivers, patient support organizations, and an online education programme.

There are few studies about anxiety or HRQoL in patients with cancer during the SARS-CoV-2 pandemic. A small study of 77 outpatients with lymphoma in one hospital reported an anxiety incidence of 36\% (Romito et al. 2020). We found that the incidence of anxiety in lymphoma patients and caregivers was about $30 \%$ and $50 \%$ higher than in normals in our survey. In our cross-sectional study, more than $70 \%$ of respondents had minimal/moderate anxiety. Covariates associated with low incidence of anxiety included no SARS-CoV-2 infection, not being a lymphoma patient or caregiver, physical exercise, higher education level, $\mathrm{a}>2 \mathrm{~h}$ increase in daily mobile phone use, and a higher family support score. Among patient respondents, physical exercise and better caregiver support were associated with less anxiety, whereas female sex, receiving therapy, and reduced therapy intensity were associated with more anxiety.

Previous cancer patient-caregiver dyads studies reported caregivers experienced similar or higher anxiety levels compared with patients (Li et al. 2018; Nipp et al. 2016; Sklenarova et al. 2015). We found a similarly increased incidence of anxiety in lymphoma patients and caregiver respondents. In China, caregivers, typically young family members, are deeply involved in patients' medical care and related activities based on the concept of filial piety often assuming responsibility for patients' financial, physical, and mental support. There were some discordances between patient co-varieties reported by patients and by their caregivers possibly reflecting some degree of technical change posed by electronic reporting by older persons. A higher proportion of patients with aggressive lymphoma reported by the caregiver compared with patient respondents is consistent with caregivers' concern for patients. Many studies report that caregivers' well-being is an important aspect for patients' mental health (Castillo et al. 2019). Consequently, we were not surprised to find patients scoring their caregiver support high had a lower incidence of anxiety compared with patients giving low scores.

We were surprised to find that HRQoL of lymphoma patients during the pandemic was better than a propensity score matched cohort before the pandemic. When we analyzed unbalanced co-variates between the two cohorts correlating with HRQoL, we found no impact of increased daily mobile phone use. A reduction in therapy intensity, however, was significantly associated with worse HRQoL. Patient respondents who scored caregiver support high had a better general HRQoL, physical and emotional function, cognitive function, and fewer or less severe symptoms of insomnia and diarrhea. The lower diarrhea score is presumably related to the perception of severity rather than incidence, frequency, or severity. Social support resources for lymphoma patients besides caregivers included online patient support/discussion groups such as House 086 and the CSCO professional education programme. We found that subjects who rated the quality of these online tools high had a better HRQoL.

Reducing cancer therapy intensity during the pandemic is not an evidence-based recommendation (Al-Shamsi et al. 2020; Di Ciaccio et al. 2020; ESMO 2020; Ismael et al. 2020). However, decreased therapy intensity was reported by $24 \%$ of patients and caregiver respondents in our survey, and was associated with a higher incidence of anxiety and worse HRQoL. Our questionnaire did not allow us to determine why therapy intensity was reduced, but limited access to medical care at times when significant resources were used to cope with the challenges of the pandemic is the most likely reason.

There are several limitations of our study. Our survey was online with potential selection biases. For example, our patient respondents were younger than most lymphoma patients, perhaps because of increased Internet familiarity and/or access. Not surprisingly, our normals cohort had a much younger age than patient and caregiver 
respondents. Because our survey was cross-sectional, it was not possible to compare anxiety and HRQoL in the same respondent before and during the pandemic. In our comparison of HRQoL with a pre-pandemic cohort, we lacked data on lymphoma stage, so we cannot know if the better HRQoL which we observed during the pandemic might result from patients with less advanced lymphoma (Stewart et al. 2016).

\section{Conclusion}

During the SARS-CoV-2 pandemic, lymphoma patients and their caregivers had a significantly higher incidence of anxiety compared with normals. Incidence was increased in persons stopping or reducing therapy intensity. Paradoxically, lymphoma respondents had a better HRQoL compared with pre-pandemic lymphoma controls. Good social services including caregiver support and an online lymphoma education programme were associated with less anxiety and better HRQoL. Reduced therapy intensity was also associated with worse HRQoL. Reduced therapy intensity in cancer patients during the SARS-CoV-2 pandemic may have negative impact on patient anxiety and HRQoL.

Acknowledgements Yinan Sun MS, Director of Student Wellness (Yungu School; China) reviewed the questionnaire. Prof. Norbert Schmitz (University Hospital, Münster, Germany) kindly reviewed the typescript. RPG acknowledges support from the National Institute of Health Research (NIHR) Biomedical Research Centre funding scheme.

Author contributions SY designed the study and the questionnaire, analyzed data, interpreted the results, and drafted the manuscript. DD conducted the 2019 lymphoma survey and extracted matched subjects from the 2019 lymphoma database. HG conducted the online surveys and collected data in pre- and during the pandemic. RPG helped to develop the typescript. JM supervised and organized the CSCO patients' education programme and designed the study. $\mathrm{XH}$ designed and supervised the study. All authors reviewed and approved the typescript.

\section{Funding None.}

Data availability The datasets used and analyzed during the current study are available from the corresponding author upon request.

\section{Compliance with ethical standards}

Conflict of interest RPG is a consultant to: BeiGene Ltd., Fusion Pharma LLC, LaJolla NanoMedical Inc., Mingsight Parmaceuticals Inc., and CStone Pharmaceuticals. Advisor: Antegene Biotech LLC, Medical Director: FFF Enterprises Inc. Partner: AZACA Inc. Board of Directors: RakFond Foundation for Cancer Research Support. Scientific Advisory Board: StemRad Ltd.

Ethical approval Approved by the Ethical Committee of Peking Univeristy Peoples Hospital (Register number 2020PHB173).
Informed consent Electronic informed consent was obtained from all respondents who could withdraw at any time during the survey without prejudice.

\section{References}

Aaronson NK, Ahmedzai S, Bergman B et al (1993) The European Organization for Research and Treatment of Cancer QLQ-C30: a quality-of-life instrument for use in international clinical trials in oncology. J Natl Cancer Inst 85(5):365-376. https://doi. org/10.1093/jnci/85.5.365

Al-Shamsi HO, Alhazzani W, Alhuraiji A et al (2020) A practical approach to the management of cancer patients during the novel coronavirus disease 2019 (COVID-19) Pandemic: An International Collaborative Group. Oncologist 25(6):e936-e945. https ://doi.org/10.1634/theoncologist.2020-0213

Cao C, Feng J, Gu H et al (2018) Distribution of lymphoid neoplasms in Northwest China: Analysis of 3244 cases according to WHO classification in a single institution. Ann Diagn Pathol 34:60-65. https://doi.org/10.1016/j.anndiagpath.2017.05.005

Castillo EG, Ijadi-Maghsoodi R, Shadravan S et al (2019) Community interventions to promote mental health and social equity. Curr Psychiatry Rep 21(5):35. https://doi.org/10.1007/s1192 0-019-1017-0

Chen-See S (2020) Disruption of cancer care in Canada during COVID-19. Lancet Oncol 21(8):e374. https://doi.org/10.1016/ S1470-2045(20)30397-1

Di Ciaccio P, McCaughan G, Trotman J et al (2020) Australian and New Zealand consensus statement on the management of lymphoma, chronic lymphocytic leukaemia and myeloma during the COVID-19 pandemic. Intern Med J 50(6):667-679. https://doi. org/10.1111/imj.14859

ESMO(2020) ESMO guidelines: Cancer patient management during the COVID-19 pandemic. https://www.esmo.org/guidelines/cance r-patient-management-during-the-covid-19-pandemic Accessed July 30, 2020.

Huang Y, Zhao N (2020) Generalized anxiety disorder, depressive symptoms and sleep quality during COVID-19 outbreak in China: a web-based cross-sectional survey. Psychiatry Res 288:112954. https://doi.org/10.1016/j.psychres.2020.112954

Ismael J, Losco F, Quildrian S et al (2020) Multidisciplinary approach to COVID-19 and cancer: consensus from scientific societies in Argentina. Ecancermedicalscience 14:1044. https://doi. org/10.3332/ecancer.2020.1044

Li Q, Lin Y, Xu Y et al (2018) The impact of depression and anxiety on quality of life in Chinese cancer patient-family caregiver dyads, a cross-sectional study. Health Qual Life Outcomes 16(1):230. https ://doi.org/10.1186/s12955-018-1051-3

Liu XC, Oda S, Peng X et al (1997) Life events and anxiety in Chinese medical students. Soc Psychiatry Psychiatr Epidemiol 32(2):6367. https://doi.org/10.1007/BF00788922

Maringe C, Spicer J, Morris M et al (2020) The impact of the COVID19 pandemic on cancer deaths due to delays in diagnosis in England, UK: a national, population-based, modelling study. Lancet Oncol 21(8):1023-1034. https://doi.org/10.1016/S1470 $-2045(20) 30388-0$

Minglu L, Fang F, Guanxi L et al (2020) Influencing factors and correlation of anxiety, psychological stress sources, and psychological capital among women pregnant with a second child in Guangdong and Shandong Province. J Affect Disord 264:115-122. https://doi. org/10.1016/j.jad.2019.11.148

Nipp RD, El-Jawahri A, Fishbein JN et al (2016) Factors associated with depression and anxiety symptoms in family caregivers of 
patients with incurable cancer. Ann Oncol 27(8):1607-1612. https ://doi.org/10.1093/annonc/mdw205

Romito F, Dellino M, Loseto G et al (2020) Psychological distress in outpatients with lymphoma during the COVID-19 Pandemic. Front Oncol 10(1270):1270. https://doi.org/10.3389/ fonc. 2020.01270

Shah MA, Emlen MF, Shore T et al (2019) Hematology and oncology clinical care during the coronavirus disease 2019 pandemic. CA Cancer J Clin. https://doi.org/10.3322/caac.21627

Shao R, He P, Ling B et al (2020) Prevalence of depression and anxiety and correlations between depression, anxiety, family functioning, social support and coping styles among Chinese medical students. BMC Psychol 8(1):38. https://doi.org/10.1186/s40359-020-00402 $-8$

Sklenarova H, Krumpelmann A, Haun MW et al (2015) When do we need to care about the caregiver? Supportive care needs, anxiety, and depression among informal caregivers of patients with cancer and cancer survivors. Cancer 121(9):1513-1519. https://doi. org/10.1002/cncr.29223

Stewart WC, Reynolds KE, Jones LJ et al (2016) The source and impact of specific parameters that enhance well-being in daily life. J Relig Health 55(4):1326-1335. https://doi.org/10.1007/ s10943-015-0076-8

Sud A, Torr B, Jones ME et al (2020) Effect of delays in the 2-weekwait cancer referral pathway during the COVID-19 pandemic on cancer survival in the UK: a modelling study. Lancet Oncol 21(8):1035-1044. https://doi.org/10.1016/S1470-2045(20)30392 $-2$

Sun J, Yang Q, Lu Z et al (2012) Distribution of lymphoid neoplasms in China: analysis of 4,638 cases according to the World Health Organization classification. Am J Clin Pathol 138(3):429-434. https://doi.org/10.1309/AJCP7YLTQPUSDQ5C
Triggle N (2020) (June 11) Coronavirus: Major disruption to cancer care revealed. BBC NEWS:Retrieved from https://www.bbc.com/ news/health-52985446.

van de Haar J, Hoes LR, Coles CE et al (2020) Caring for patients with cancer in the COVID-19 era. Nat Med 26(5):665-671. https://doi. org/10.1038/s41591-020-0874-8

Wang C, Pan R, Wan X et al (2020) Immediate psychological responses and associated factors during the initial stage of the 2019 coronavirus disease (COVID-19) epidemic among the general population in China. Int J Environ Res Public Health 17(5):1729. https://doi. org/10.3390/ijerph17051729

Yang QP, Zhang WY, Yu JB et al (2011) Subtype distribution of lymphomas in Southwest China: analysis of 6,382 cases using WHO classification in a single institution. Diagn Pathol 6:77. https://doi. org/10.1186/1746-1596-6-77

Zhao H, Kanda K (2000) Translation and validation of the standard Chinese version of the EORTC QLQ-C30. Qual Life Res 9(2):129-137. https://doi.org/10.1023/a:1008981520920

Zung WW (1971) A rating instrument for anxiety disorders. Psychosomatics 12(6):371-379. https://doi.org/10.1016/S0033 $-3182(71) 71479-0$

ZY W (1984) Chinese version of Zung's self-rating anxiety scale. J Shanghai Psychiatry 2:73-74

Publisher's Note Springer Nature remains neutral with regard to jurisdictional claims in published maps and institutional affiliations. 